\title{
Improvement of the grand canonical quantum Monte Carlo method at low temperatures
}

\author{
M. Ulmke and H. Müller-Krumbhaar \\ Institut für Festkörperforschung, KFA Jülich, W-5170 Jülich, Federal Republic of Germany
}

Received September 19, 1991

We introduce an improvement of the algorithm for the simulation of correlated fermi systems in the grand canonical ensemble. Using this new method the computer time grows no more with the square but essentially linearly with the inverse temperature. At low temperatures the number of operations is diminished by a factor typically between 5 and 20 . We present results for correlation functions of the one-dimensional Hubbard model at various band fillings.

\section{Introduction}

Computer simulations have proven to be a powerful tool for the study of correlated fermi systems. In contrast to almost all analytic techniques in this field they contain no uncontrolled approximations. On the other hand, they allow one to investigate much larger systems than by exact diagonalisation and to draw conclusions for the thermodynamic limit.

The algorithm for finite temperature studies of grand canonical ensembles has been basically developed by Blankenbecler et al. [1], and by Hirsch [2], who applied this method for the first time to the Hubbard model.

The major problem in this algorithm is to reach sufficiently low temperatures. The energy scale of the interesting phenomena, such as magnetism or superconductivity, is typically 100 times smaller than the bandwidth. Since the fermi matrix has eigenvalues between one and about $\exp (\beta E)$ ( $\beta$ is the inverse temperature, $E$ the bandwidth), the wide range of eigenvalues at low temperatures causes numerical instabilities. This problem has been removed by using matrix-decomposition methods which make simulations at very low temperatures possible $[3,4,5]$.

Simulations of many-fermion systems require a large amount of computer time, because the number of operations grows cubically with the system volume, $V$, and, at low temperatures, quadratically with $\beta$. It is due to the fact that the fermion matrix has to be recomputed periodically from scratch which requires a large number (proportional to $\beta$ ) of matrix multiplications. The $\beta^{2}$ dependence makes this algorithm unsuituable for simulations at very low temperatures. In this paper we show that one can reduce the $\beta$-dependence from $\beta^{2}$ to $\beta \ln \beta$ by storing and updating the intermediate products of matrices in a hierarchical structure. This method diminishes the number of operations by a factor of typically between 5 and 20. In Sect. II we give a brief description of the algorithm and explain the new method. We refer to [5] and use their notation. In Sect. III we present results for correlation functions of the Hubbard model in one dimension at various band fillings and compare the spatial decay with results from renormalization-group theory.

\section{The algorithm}

The algorithm can be applied to a variety of many-fermion models. Here we illustrate it by the example of the Hubbard model $[8,9,10]$. The present description is very compressed, for more detailed explanation see the given references, in particular [5]. The Hubbard hamiltonian can be written in the form $H=K+V$ with

$$
\begin{aligned}
K= & -t \sum_{\langle i, j\rangle, \sigma}\left(c_{i \sigma}^{+} c_{j \sigma}+\text { h.c. }\right) \\
& -\mu \sum_{i}\left(n_{i+}+n_{i-}\right) \equiv \sum_{i, j, \sigma} c_{i \sigma}^{\dagger} k_{i j} c_{j \sigma}, \\
V= & U \sum_{i}\left(n_{i+}-\frac{1}{2}\right)\left(n_{i-}-\frac{1}{2}\right) .
\end{aligned}
$$

$c_{i \sigma}^{\dagger}$ and $c_{i \sigma}$ are creation and anihilation operators for electrons with spin $\sigma$ at lattice site $i, n_{i \sigma}=c_{i \sigma}^{\dagger} c_{i \sigma}$. The sum $\langle i, j\rangle$ runs over all pairs of nearest neighbor lattice sites. $\mu$ is the chemical potential, $U$ the on-site Coulomb coupling and $t$ the hopping parameter which we define as energy scale. 
Using the Trotter-Suzuki decomposition [6] and a discrete Hubbard-Stratonovich transformation [7] which introduces an auxiliary field of Ising spins, the grand partition function $Z=\operatorname{Tr}\left[\mathrm{e}^{-\beta H}\right]$ can be integrated out for fixed Ising field yielding:

$Z=\sum_{S_{i, l}= \pm 1} \operatorname{det} M^{+} \operatorname{det} M^{-}$.

The sum runs over all configurations of the Ising spins $s_{i, l}= \pm 1, i$ is a lattice site, $l=1, \ldots, L$ is the index of the Trotter step or imaginary time slice. $L=\beta / \Delta \tau$ is the number of time slices, and $\Delta \tau$ the size of an imaginary time step.

The fermion matrices are given as

$$
\begin{aligned}
& M^{\sigma}=I+B_{L}^{\sigma} B_{L-1}^{\sigma} \cdots B_{1}^{\sigma}, \\
& B_{l}^{\sigma}=\mathrm{e}^{-\Delta \tau \sigma \lambda v(l)} \mathrm{e}^{-\Delta \tau k} .
\end{aligned}
$$

$I$ is the $V \times V$ unit matrix, $V$ the number of lattice sites, $k$ the matrix defined in (1) and $v(l)_{i j}=\delta_{i j} s_{i, l} . \lambda$ is defined by the relation $\cosh (\Delta \tau \lambda)=\exp (\Delta \tau U / 2)$. Thus, the problem is mapped onto a classical $d+1$-dimensional system of Ising spins. The summation in (3) is performed employing the Monte Carlo scheme. However, the product of determinants in (3) is in general not positive definite and therefore not a suitable statistical weight. In such a case one consider the absolute value of the summand as weight, and the sign occurs in the determination of observables (see e.g. [5]). In the positive $U$ Hubbard model in $d \geqq 2$, away from half filling, the average sign goes exponentionally to zero with $\beta$ and $V$. This causes large cancellations enforcing very long simulation runs to reach a sufficient statistical accuracy. In $d=1$ this so called sign problem does not occur for any band filling. We obtain the one particle Green's function for electrons propagating through the field of Ising spins at imaginary time $l \Delta \tau$ through

$G^{\sigma}(l)=\left[I+B_{l}^{\sigma} B_{l-1}^{\sigma} \cdots B_{1}^{\sigma} B_{L}^{\sigma} B_{L-1}^{\sigma} \cdots B_{l+1}^{\sigma}\right]^{-1}$.

Any physical observable, $O$, can now be calculated from $G^{\sigma}(l)$.

Using the specific shape of (5) and (6), the change of the equal time Green's function $G^{\sigma}(l)$ and the statistical weights under a single spin-flip, $s_{i, l} \rightarrow-s_{i, l}$, can be calculated by a "local" procedure, i. e. it takes $V^{2}$ operations to update the $V \times V$ matrix $G^{\sigma}(l)$. After updating $G^{\sigma}(l)$, the Green's function for the next time slice can be obtained by

$G^{\sigma}(l+1)=B_{l+1}^{\sigma} G^{\sigma}(l)\left(B_{l+1}^{\sigma}\right)^{-1}$.

However, this propagation introduces numerical errors after some number $m$ of time slices which enforces a periodical recomputation of $G^{\sigma}(l)$ from (6). The fermion matrix has eigenvalues over a broad range in magnitude $(\sim \exp (\beta E))$. Thus, at low temperatures, information about the small eigenvalues gets lost due to of round-off errors. This problem can be dealt with using matrix orthogonalization methods as described in [5]. A number $m$ (as before) of matrices $B_{l}^{\sigma}$ can be multiplied with each other without numerical problems before a decomposition of the product is necessary. Note that this factorization works in (6) but not in (7). We used an $U R$ decompostion by Householder transformations ( $U$ is orthogonal, $R$ an upper triangular matrix). Afterwards we scale the rows of $R$ so that the diagonal elements become equal to one. Now we can write the matrix in the form $U D R^{\prime}$, where the diagonal matrix $D$ contains the former diagonal of $R$. We found that this factorization method takes about 1.5 times as many operations as an ordinary matrix multiplication. Altogether the following operations are required for a Monte Carlo sweep through $m$ time slices - i.e. each Ising spin is tested for a spin-flip once in each of $m$ time slices $(Q \equiv L / m)$ :

- $m$ updates according to (7)

- $L$ multiplications of matrices $B_{l}^{\sigma}$ according to (6)

- $Q$ factorizations and multiplications of triangular matrices

So the whole number of matrix multiplications and equivalent processes for a complete sweep of the Ising field is about

$\Sigma=Q(2 m+L+1.7 Q)$

Since $Q$ and $L$ are proportional to $\beta$, the computing time grows quadratically with $\beta$ for low temperatures.

The spinflips in one single time slice, $l$, does not effect any matrix in the product (4) besides $B_{l}^{\sigma}$. Nevertheless after $m$ updatings by (7) the complete product of all matrices $B_{l}^{\sigma}$ including the decompositions must be performed to obtain $G^{\sigma}(l)$. This can be avoided by storing intermediate products in a hierarchical structur as we will now explain.

At the beginning we perform products of $m$ matrices $B_{l}^{\sigma}$ each:

$P_{k}^{\sigma}=B_{m \cdot k}^{\sigma} B_{m \cdot k-1}^{\sigma} \cdots B_{m \cdot(k-1)+1}^{\sigma}$

$(k=1, \ldots, Q)$,

factorize and store them in the decomposed $U D R$-form. Then we multiply these products two by two and store the results. This is the first level of branches of this hierarchical tree. The procedure is repeated until, after $\log _{2}(Q)$ steps, the whole product

$A^{\sigma}(L)=P_{Q}^{\sigma} P_{Q-1}^{\sigma} \cdots P_{1}^{\sigma}=B_{L}^{\sigma} B_{L-1}^{\sigma} \cdots B_{1}^{\sigma}$

is performed and the equal time Green's function can be calculated as described in [5]. Thus, a hierarchical tree of matrix products is created.

The multiplication of two factorized matrices goes as follows:

$$
\begin{aligned}
U_{2}\left(D_{2} R_{2}\right)\left(U_{1} D_{1}\right) R_{1} & =U_{2}\left(R_{2}^{\prime} U_{1}^{\prime}\right) R_{1} \\
& =U_{2}(U D R) R_{1}=U_{21} D_{21} R_{21}
\end{aligned}
$$

First we multiply the diagonal matrices $D_{2}$ and $D_{1}$ with $R_{2}$ and $U_{1}$, resp. Then we multiply the results with each other, decompose the product and multiply the two orthogonal and the two triangular matrices, resp., with each 
other. The result is thus $U D R$-factorized. This procedure is equivalent to about three matrix multiplications concerning computer time, if one uses the triangular shape of the $R$-matrices. After $m$ updates by (7) one has to calculate $G^{\sigma}(m)$ from scratch. For that pupose the product

$$
\begin{aligned}
A^{\sigma}(m) & =P_{1}^{\sigma} P_{Q}^{\sigma} \cdots P_{2}^{\sigma} \\
& =B_{m}^{\sigma} B_{m-1}^{\sigma} \cdots B_{1}^{\sigma} B_{L}^{\sigma} B_{L-1}^{\sigma} \cdots B_{m+1}^{\sigma}
\end{aligned}
$$

is required. Since until then only Ising spins of the first $m$ time slices have been flipped, $P_{1}^{\sigma}$ is the only matrix which has been changed. Then $A^{\sigma}(m)$ can be determined by the stored matrix products, where in each of the $\log _{2}(Q)$ levels only one new matrix product has to be evaluated. In other words, instead of performing all products (12) we have to recompute only the products at the $\log _{2}(Q)$ nodes in the hierarchical tree which are crossed when moving from one outer branch to the stam. Employing this algorithm, the following operations are required for a Monte Carlo sweep through $m$ time slices:

- $m$ updates according to (7)

- calculation of $P_{k}^{\sigma}(k=1, \ldots, Q)$, according to (9)

- $\log _{2}(Q)$ multiplications of factorized matrices

For a complete sweep of the Ising field this procedure has to be repeated analogously $Q$ times. Therefore the number of matrix multiplications (and equivalents) is:

$\Sigma^{\prime}=Q\left[3 m+3 \log _{2}(Q)\right]$

For typical values of the parameters $(\Delta \tau=1 / 8$, $L=\beta / \Delta \tau, m=8)$ and inverse temperatures $\beta=32,64$ and 128 the relation $\Sigma / \Sigma^{\prime}$ becomes 8,15 and 28 resp. Since at very low temperatures $(\beta \geq 60)$ the calculation of the Green's function consumes more than $90 \%$ of the operations, the effect on the computer time request is substantial. The reduction of CPU-time consumption is bought with an increase of required memory capacity. Two times (spin up/down) $3 \mathrm{~L} / \mathrm{m}$ factorized $V \times V$-matrices have to be stored, i.e. about $9 \mathrm{~L} / \mathrm{m} \cdot V^{2}$ real numbers. For rather large systems at very low temperatures - e.g. $V=100, \beta=128$ - the required memory capacity is about 100 MByte (with 64-Bit precision).

The algorithm looses nothing of its generality and can be applied to a variety of fermion models. In the case of the one band Hubbard model the $B_{l}^{\sigma}$ are essentially sparse and so the operations (4) and (7) take of the order of $V^{2}$ multiplications. Therefore the number of $V^{3}$-processes is drastically reduced and the ratio between the number of $V^{3}$-processes in the two algorithms decreases to $3.6,6$ and 10 for the inverse temperatures above.

We tested the algorithm for the one band Hubbard model in two and three dimensions in the half filled band case and in one dimension at different band fillings. For a Coulomb coupling $U \leqq 8$ the algorithm is numerically stable for inverse temperatures tested up to $\beta=128$. The simulations have been performed on a CRAY X-MP at the Forschungszentrum Jülich. At an inverse temperature of $\beta=32$ a complete sweep of the Ising field takes $3.5 \mathrm{~s}$ for $V=32$ and $50 \mathrm{~s}$ for $V=100$ (with parameters
$\Delta \tau=0.125$ and $m=8$ ). The computing time grows linearly with $\beta$ but does not scale with $V^{3}$ because of the better vectorisation at $V=100$. The performance speed in the latter case is slightly above 100 MFlops. For the simulation typically 500 warm-up sweeps and 2000 measurements separated by two sweeps were performed for each set of parameters.

\section{Results}

In the Hubbard model each site can be either empty or occupied by one or two electrons. We define the corresponding correlation functions through the following sums over lattice sites:

$C_{j}^{X Y} \equiv \frac{1}{V} \sum_{i=1}^{V}\left\langle X_{i} Y_{i+j}\right\rangle-\langle X\rangle\langle Y\rangle$.

$X_{i}$ and $Y_{i}$ are two of the following operators: $S_{i} \equiv n_{i \uparrow}-n_{i \downarrow}$ (spin), $\quad N_{i} \equiv n_{i \uparrow}+n_{i \downarrow}$ (electron density), $D_{i} \equiv n_{i \uparrow} n_{i \downarrow}$ (double occupation) and $H_{i} \equiv\left(1-n_{i \uparrow}\right)\left(1-n_{i \downarrow}\right)$ (density of empty sites, here called holes for convenience). The mean densities $(s, n, d, h)$ are given as

$x \equiv\langle X\rangle=\frac{1}{V} \sum_{i=1}^{V}\left\langle X_{i}\right\rangle$.

Only four of the ten possible correlation functions are independent. It is therefore sufficient to calculate the four functions $C_{j}^{X X}$. We investigated in detail the spatial decay of spin-spin and density-density correlation functions. According to renormalization-group (RG) theory [11, 12], a long-range power-law behaviour with additional logarithmic corrections is expected: $C_{j}^{X X} \sim j^{-y}$. Schulz [13] determined the correlation exponents, $\gamma$, for the one-dimensional Hubbard model for arbitrary band filling and Coulomb interaction strength. In Fig. 1 and 2 Monte Carlo data for $C_{j}^{S S}$ and $C_{j}^{N N}$ respectively are shown in a double logarithmic plot. Data are plotted for the ring

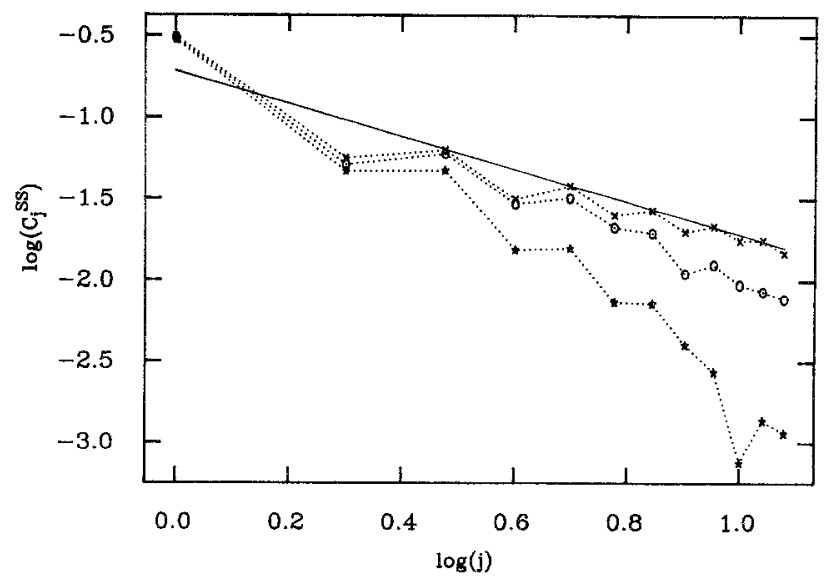

Fig. 1. Spatial decay of the spin-spin correlation, $C_{j}^{s s}$, for $V=32$, $U=2, n=1.0 ; *: \beta=8,0: \beta=16, \times: \beta=32$. Solid curve: straight line with slope -1 


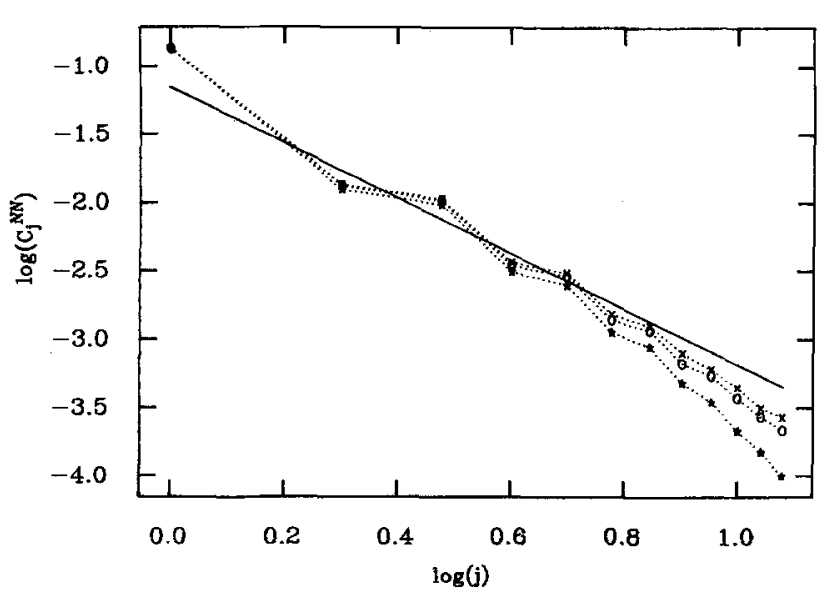

Fig. 2. Spatial decay of the density-density correlation, $C_{j}^{N N}$, for $V=32, U=2, n=1.0 ; *: \beta=8, \circ: \beta=16, \times: \beta=32$. Solid curve: straight line with slope -2

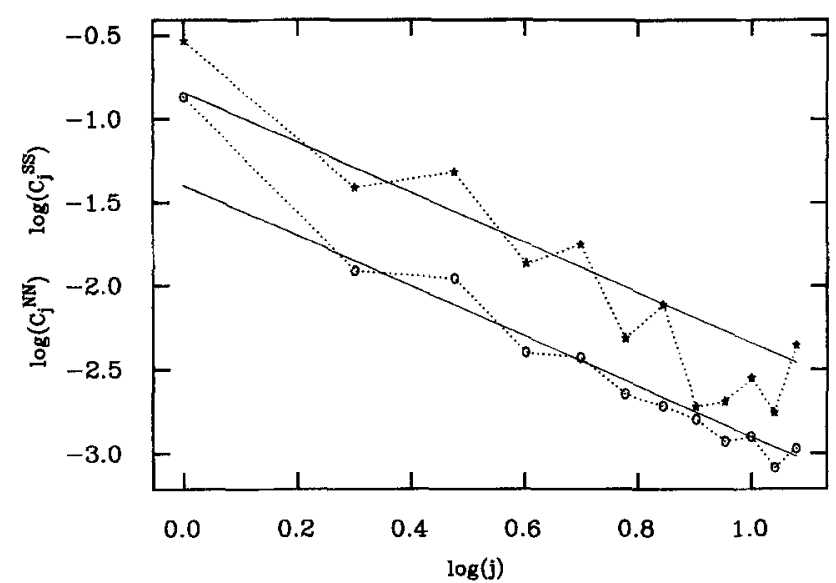

Fig. 3. Spatial decay of $C_{j}^{S S}$ (above) and $C_{j}^{N N}$, for $V=32, U=2$, $n=0.94, \beta=32$. Solid curves: straight lines with slope -1.5

with 32 sites and $U=2$ at half filling $(n=1.0)$. The error bars, estimated by the results of several independent runs, are of the size of the symbols. Indeed, the power law decay becomes visible for decreasing temperature. Although at finite temperatures one should expect an exponential decay, the results become undiscriminable from power-law decay, when the thermal correlation length is larger than the system size. This seems to be the case for $\beta=32$, since the correlation functions do not alter significantly for temperatures below this value. On the other hand, the convergence to the thermodynamic limit seems to be quite good for $V=32$. For $C_{j}^{S S}$, the exponent $\gamma$ lies quite close to unity, corresponding to the antiferromagnetic ordering of the electron spins and in agreement to [13], where $\gamma=1$ is predicted for $C_{j}^{S S}$ as well as for $C_{j}^{N N}$. In contrast to this, $\gamma$ seems to converge to a value of two for $C_{j}^{N N}$ in the simulation. This might be due to an additional $j^{-2}$-term which dominates at short distances, or to logarithmic corrections.

According to RG theory, $\gamma$ is always larger than one for $n \neq 1$. It is interesting that $\gamma$ does not converge to unity as $n$ converges to half filling. Instead, a value of

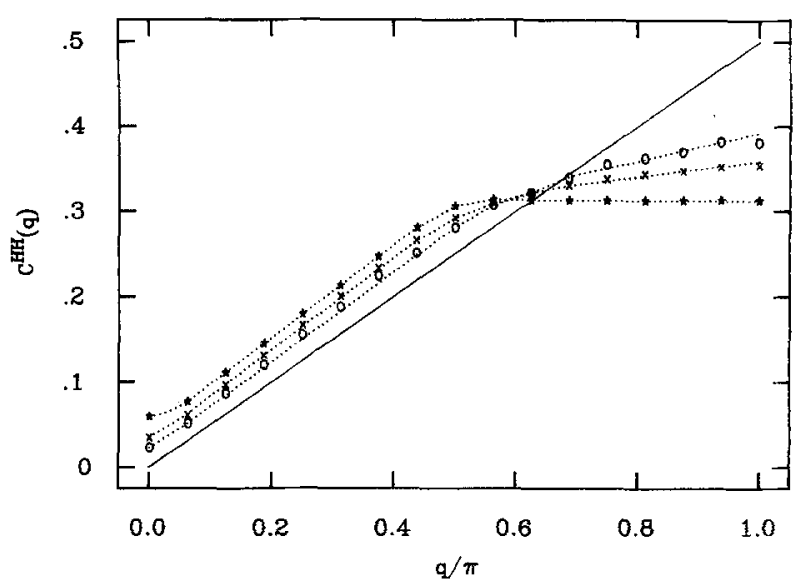

Fig. 4. Fourier transform of the hole-hole correlation, $C^{H H}(q)$, for $V=32, n=0.5, \beta=32 ; *: U=0, \times: U=2,0: U=4$. Solid curve: exact result for $U=\infty$ for the infinte system at $T=0$. (Dotted curves are drawn to guide the eye)

$\gamma=1.5$ is predicted in the close proximity of $n=1$ for arbritary values of $U[13]$. Monte Carlo results for $n=0.94$ at $U=2$ confirm this value for both $C_{j}^{S S}$ and $C_{j}^{N N}$ (Fig. 3).

Figure 4 finally shows the fourier transform of the hole-hole correlation function, $C^{H H}(q)$, for $U=0,2$ and 4 for the 32-site ring at quarter band filling. The solid line depicts the exact result for $U=\infty$ for the infinite chain. In this (atomic) limit, each site is either empty or singly occupied, so the holes can move independently and behave like spinless, free fermions. For $U=0$ the curve has a slope equal to the density $h$ for $q<n \pi$ and becomes horizontal at this wave vector due to Umklapp-processes. The transition of $C^{H H}(q)$ between these limits is clearly visible. For $U<\infty$ the number of holes is not conserved, thus the value $C^{H H}(q=0)$ which is $V$-times the variance of hole-density does not vanish.

\section{Conclusions}

In summary, we have shown how one can reduce the temperature-dependence of computing time in the grand canonical quantum Monte Carlo algorithm from $\beta^{2}$ to $\beta \ln \beta$. At low temperatures the computing time can be reduced by a factor of practically up to 20 . Employing this method, the storage request increases to typically 10 to 100 MByte. We applied the method to the Hubbard model and calculated correlation functions for one-dimensional systems. The spin-spin and density-density correlations for $n=1.0$ and 0.94 exhibit a power-law decay. Except for the density-density correlations at $n=1.0$, where probably the short range behaviour is still dominant, the correlation exponents agree with the renormalization-group results.

We would like to thank D. Vollhardt and M. Frick for helpful discussions and suggestions. 


\section{References}

1. Blankenbecler, R., Scalapino, D.J., Sugar, R.L.: Phys. Rev. D24, 2278 (1981); Scalapino, D.J., Sugar, R.L.: Phys. Rev. B24, 4295 (1981)

2. Hirsch, J.E.: Phys. Rev. B31, 4403 (1985)

3. Koonin, S.E., Sugiyama, G., Friedrich, H.: In: Proceedings of the International Symposium Bad Honnef. Goeke, K., Greinhard, P.G. (eds.), p. 214. Berlin, Heidelberg, New York: Springer 1982

4. Sorella, S., Tosatti, E., Baroni, S., Car, P., Parinello, M.: In: Proceedings of the Adriatico Research Conference "Towards the theoretical understanding of the high- $T_{c}$ Superconductors". Lundquist, S., Tosatti, E., Tosi, M.P., Lu, Y. (eds.), p. 457. Singapore: World Scientific 1988

5. White, S.R., Scalapino, D.J., Sugar, R.L., Loh, E.Y., Gubernatis, J.E., Scalettar, R.T.: Phys. Rev. B40, 506 (1989)

6. Suzuki, M.: Prog. Theor. Phys. 56, 1454 (1976)

7. Hirsch, J.E.: Phys. Rev. B25, 4059 (1983)

8. Gutzwiller, M.C.: Phys. Rev. Lett. 10, 159 (1963)

9. Kanamori, J.: Prog. Theor. Phys. 30, 275 (1963)

10. Hubbard, J.: Proc. R. Soc. London A 276, 283 (1963)

11. Sólyom, J.: Adv. Phys. 28, 209 (1979)

12. Giamarchi, T., Schulz, H.J.: Phys. Rev. B39, 4620 (1989)

13. Schulz, H.J.: Phys. Rev. Lett. 64, 2831 (1990) 